Document downloaded from:

http://hdl.handle.net/10251/83532

This paper must be cited as:

Martínez Pinzón, G.; Cardona Marcet, N.; García Pardo, C.; Fornés Leal, A.; RibadeneiraRamírez, JA. (2016). Spectrum Sharing for LTE-A and DTT: Field Trials of an Indoor LTE-A Femtocell in DVB-T2 Service Area. IEEE Transactions on Broadcasting. 62(3):552-561. doi:10.1109/TBC.2016.2582338.

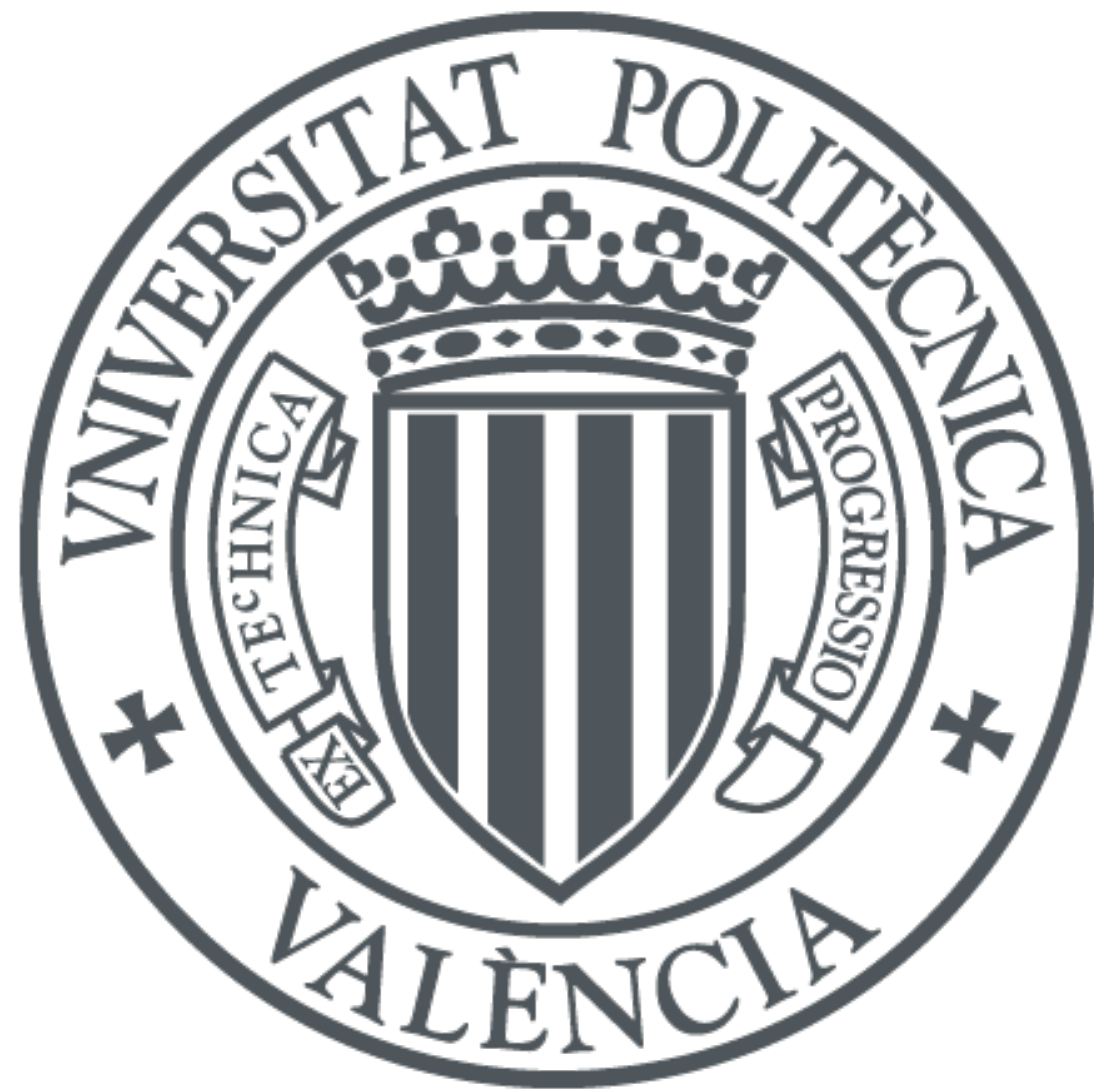

The final publication is available at

http://dx.doi.org/10.1109/TBC.2016.2582338

Copyright Institute of Electrical and Electronics Engineers (IEEE)

Additional Information

(c) 2016 IEEE. Personal use of this material is permitted. Permission from IEEE must be obtained for all other users, including reprinting/ republishing this material for advertising or promotional purposes, creating new collective works for resale or redistribution to servers or lists, or reuse of any copyrighted components of this work in other works. 


\title{
Spectrum Sharing for LTE-A and DTT: Field Trials of an Indoor LTE-A Femtocell in DVB-T2 service area
}

\author{
G. Martínez-Pinzón, N. Cardona, C. Garcia-Pardo, A. Fornes-Leal and J. Ribadeneira-Ramirez
}

\begin{abstract}
In the near future, many applications such as environmental sensors, smart objects, health sensors and personal devices will be connected to mobile networks requiring additional spectrum. Studies have been made to demonstrate a low occupancy time and locations on the DTT band. This available or unused spectrum have been called "TV-WhiteSpaces". In the 2015 World Radiocommunication Conference, the ITU decided to re-allocate the $700 \mathrm{MHz}$ band $(694-790 \mathrm{MHz})$ for IMT services and also emplaced the discussion of the future use of the DTT band (470-694 MHz) to 2025, for which studies have been requested.

In this paper we study a particular case which goes a step beyond the previous ones, as it aims at sharing the same frequency band in the same area between Long Term Evolution Advance (LTE-A) and Digital Video Broadcasting - Terrestrial Second generation (DVB-T2) technologies. Those geographical areas that are not covered because the useful DTT signal is obstructed by the environment or it has a limited coverage by the network design can be called "micro-TVWS" ( $\mu$ TVWS). We assume that a DVB-T2 transmitter provides coverage for fixed rooftop reception as a primary service, to a building in which a LTE-A femtocell is installed indoors for local coverage, as a secondary service. The results have been obtained by laboratory emulation and validated through field measurements using professional equipment. Our results provide the technical restrictions of the LTE-A Femtocell, mainly on the maximum allowable Effective Isotropic Radiated Power (EIRP) that could transmit on the DTT band in terms of carrier separation, from co-channel to adjacent band. These results meet the need of spectrum for IMT-Advanced technologies, so spectrum sharing is proposed in this paper as a new solution to make an efficient use of this resource.
\end{abstract}

Index Terms - DVB-T2, LTE-A Femtocell, White Space, Spectrum Sharing, Digital Terrestrial Television, IMT-Advance Technologies.

\section{INTRODUCTION}

$\mathrm{T}$ he increasing demand of applications and scenarios that converge on the massive use of mobile communications has led to a need for additional spectrum allocation. New applications such as environmental sensors, smart objects,

Manuscript received December 22, 2015; reviewed May 6, 2016; accepted June 7, 2016. This work was supported by the Ministerio de Educación y Ciencia, Spain, ("DEFINE5G" TEC2014-60258-C2-1-R and "ARCO5G" TEC2014-56469-REDT), by the European FEDER funds.

The authors are with the iTEAM Research Institute, Universitat Politècnica de València, 46022 Valencia, Spain (email: \{gemarpin, ncardona, cgpardo, alforlea, jefrira\} @iteam.upv.es). Jefferson Ribadeneira is also with the Escuela Superior Politécnica de Chimborazo (ESPOCH), Riobamba, Ecuador. (email: jefferson.ribadeneira@espoch.edu.ec). health sensors and personal devices will be connected through mobile devices, so an exponential increase in data traffic accessing mobile radio links is expected. The majority of reports published on actual mobile traffic profiles claim that about $50 \%$ of voice calls and $70 \%$ of the data traffic is generated in indoor scenarios [1]. Cisco Visual Networking Index (VNI) considered that global mobile data traffic will be multiplied by 10 in the next five years, reaching 30.6 Exabytes per month in 2020 (3.7 Exabytes in 2015), representing an annual increase rate of 53 percent from 2015 to 2020 [2]. Furthermore, The International Telecommunication Union (ITU), in its report ITU-R M.2290, estimated that $1960 \mathrm{MHz}$ radio spectrum will be necessary by 2020 to allow the appropriate development of technologies International Mobile Telecommunications (IMT) 2000 and Advanced [3].

Since radio spectrum is a natural, scarce and highly demanded resource for the use of wide range wireless technologies, governments and international organizations are considering new and more flexible solutions to make a more efficient use of it. The ITU decided to re-allocate the upper part of the Digital Terrestrial Television (DTT) band (from 790 to 862 ) for IMT technologies in the so called Digital Dividend (DD1) and announced their intentions to allocate more spectrum for mobile broadband use (700 $\mathrm{MHz}$ band) [4]. For this purpose, many coexistence studies evaluating the interference of LTE signals into DTT services have been driven. To quantify the interference problem, protection ratios were calculated for several LTE configurations, located in the lower part of the assigned band, for both downlink and uplink cases. In [5] protection ratio measurements for DVB-T2 interfered by LTE for both downlink and uplink, and link budget analysis were presented. In [6] and [7] protection ratio measurements for LTE interfering to DVB-T and DVB-T2 were made. In [8] and [9], possible solutions are proposed to mitigate interference problems between DTT and LTE in adjacent channel, such as increasing the guard band or use low pass filters. After considering several coexistence scenarios and using the measured protection ratios, some planning studies were performed [10]. In [11], the coexistence between an indoor LTE femtocell and a DVB-T2 Lite in the 800 band is proposed. In this study, a linear model to evaluate the impact of coexistence on performance of both systems is suggested. Lamy's report recommended to re-allocate the 700 MHz band for IMT technologies around 2020[12]. In addition, it proposed the ratification of the $470-694 \mathrm{MHz}$ frequency band for DTT as a primary service, with the possibility of 
using this band for additional downlink broadband services (always accomplishing the quality of the primary service).

The Electronic Communications Committee (ECC) considers four classes of long-term scenarios for the 470-694 $\mathrm{MHz}$ band [13]: Primary usage of the band by existing and future Digital Video Broadcasting (DVB); Hybrid usage of the band by DVB and/or downlink LTE terrestrial networks (LTE SDL/eMBMS); Hybrid usage of the band by DVB and/or LTE including uplink; Future communication technologies. Therefore, in the 2015 World Radiocommunication Congress (WRC-15), the ITU decided to re-allocate the $700 \mathrm{MHz}$ band (694-790 MHz) for IMT services and also emplaced the discussion of the future use of the DTT band $(470-694 \mathrm{MHz})$ to 2025 , for which studies have been requested [14].

Furthermore, other studies have been made to optimize the use of the spectrum in the UHF band. In this way, some studies have been conducted to demonstrate a low occupancy time and location on the DTT band. A study carried out in 11 countries in Europe in the 470-790 MHz band concluded that approximately $56 \%$ of the TV channels are unused, when averaged over a whole geographic area [15]. These available or unused spectrum have been called "TV-White-Spaces" (TVWS) and could be used by low power devices from not licensed users, transmitting on the same or adjacent frequency. But TVWS are considered in two dimensions (time and location), and these studies have been limited to identify surface areas where a certain TV channel is unused.

In this paper we go a step beyond coexistence, since the aim is to exploit the unused radio spectrum in those geographical zones that are not covered because the useful signal is obstructed by the environment or it has a limited coverage by the network design. Those areas can be called "micro-TVWS" ( $\mu$ TVWS). The most representative cases of our definition of $\mu$ TVWS are those indoor environments in areas where DTT received power is below the required sensitivity because a TV channel is broadcasted to rooftop reception. The proposed scenario considers a DTT network offering fixed rooftop reception as a primary service, and a LTE-A femtocell giving coverage within the interior of a home or office as a secondary service. We have considered a FDD system (Frequency Division-Duplexing system) for the LTE-A service in which downlink is provided in a TV-white-space, whereas uplink would be supplied in an upper band (e.g., $700 \mathrm{MHz}$ band). Thus, the LTE-A femtocell can use a $\mu$ TVWS inside the DTT band. The advantage of this scenario is that the path loss from the femtocell to the DTT antenna are considered as a gain to determine the Maximum Equivalent Isotropic Radiated Power (EIRP) that could transmit the LTE-A femtocell. We have chosen for our studies the DVB-T2 standard. DVB-T2 is the current state-of-the art DTT system and the most adopted and deployed in the world. The main objective of this work is to establish the maximum allowable EIRP for a LTE-A Femtocell that could transmit on the same band as the DTT, in terms of the carrier separation, going from co-channel to adjacent band. To achieve this goal, we have performed measurements in laboratory conditions and we have validated these measurements with field trials, using professional equipment. These result meet the need of spectrum for IMTAdvanced technologies, so spectrum sharing is proposed in this paper as a new solution to make an efficient use of this resource. Also, it can be interesting for the ITU such as a possible application to discuss the future use of the DTT band, for regulatory entities, mobile operators and broadcasters.

The rest of the paper is structured as follows: In Section II, the proposed scenarios and the use cases are presented. In section III, the methodology of laboratory and field measurements carried out to find the maximum EIRP transmitted by a LTE-A Femtocell is explained. In Section IV, the results to establish the maximum allowable EIRP for a LTE-A Femtocell that could transmit on the same band as the DTT are presented. Finally, conclusions and future work are outlined in Section V.

\section{DTT \& LTE-A SCENARIOS}

The scenarios under analysis considers a DVB-T2 network offering fixed rooftop reception as a primary service, and a LTE-A femtocell giving coverage within the interior of a home or office as a secondary service. The DVB-T2 signal is interfered by a LTE-A DL signal (Downlink from femtocell to LTE-A users). The LTE-A femtocell uses a $\mu$ TVWS inside the DTT band. Assuming that the path loss are the lowest possible between the path from the femtocell to the receiving antenna DTT, three different configurations or scenarios have been considered as the worst cases as depicted in Fig. 1.

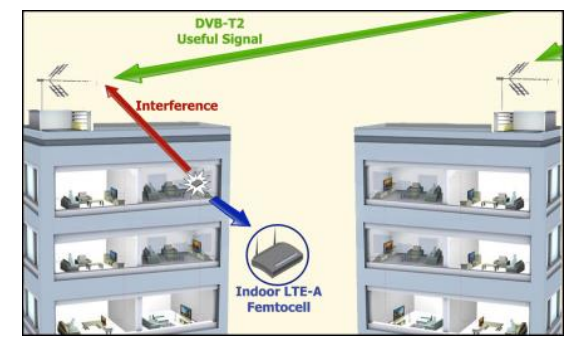

(a)

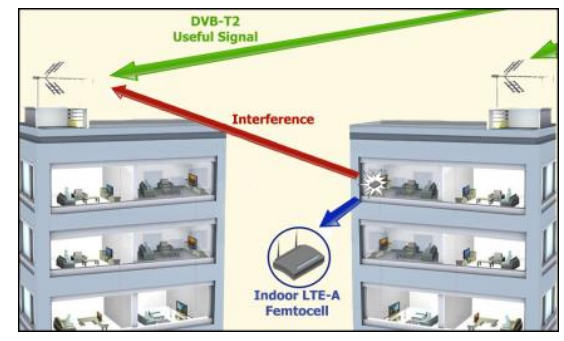

(b)

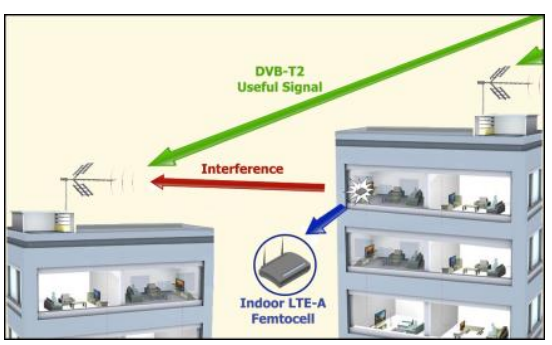

(c)

Fig 1 Evaluated scenarios for spectrum sharing between DTT and LTE-A in the DTT band. a) Scenario 1, b) Scenario 2 and c) Scenario 3. 
- Scenario 1: A femtocell is located on the top floor of the building and a DVB-T2 antenna is situated on the rooftop the same building (See Fig. 1a).

- Scenario 2: A femtocell is located on the top floor of the building and a DVB-T2 antenna is situated on the rooftop of another building. The two buildings are one next to the other, with line-of-sight (LoS) between the antennas and glass in the middle of the path (See Fig. 1b).

- Scenario 3: It's the same configuration that scenario two, but in this scenario the femtocell and the receiving antenna are located at the same height (See Fig. 1c).

For the proposed scenarios the use of existing $\mu$ TVWS in indoor is evaluated, considering the two most restrictive cases:

- Case A: Co-channel and adjacency channel: The LTE-A femtocell can use a co-channel or an adjacent channel to the DVB-T2 channel. The femtocell transmitting restrictions are evaluated in order to determine the minimum frequency spacing between central carriers from LTE-A to a DTT channel, as shown in Fig.2. We have considered that the DVB-T2 signal uses DTT channel $48(686-694 \mathrm{MHz})$ and the LTE-A DL signal transmits in co-channel or adjacency in channel 49 (694 - $702 \mathrm{MHz})$. The LTE-A central carrier is moved from co-channel to adjacent channel with respect to the DVB-T2 central carrier in steps of $0.5 \mathrm{MHz}$ to evaluate the restrictions mentioned above as shown in section 4 .

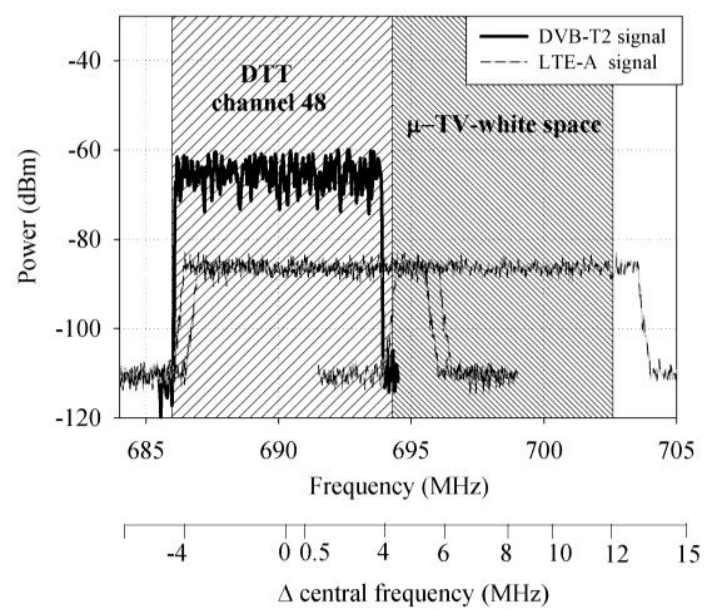

Fig 2. Case A: LTE-A Femtocell (10 MHz bandwidth) uses a $\mu$ TVWS located co-channel or adjacent channel to the DVB-T2 channel.

- Case B: Double Adjacency: The LTE-A femtocell central carrier is set between two DVB-T2 channels. Fig. 3 shows the initial configuration, in which the DVB-T2 signals are occupying the $48(686-694 \mathrm{MHz})$ and $50(702-710$ $\mathrm{MHz}$ ) channels and the LTE-A DL signal is using channel $49(694-702 \mathrm{MHz})$. In this case, the LTE-A central carrier is moved between two DVB-T2 channels in steps of $0.5 \mathrm{MHz}$.

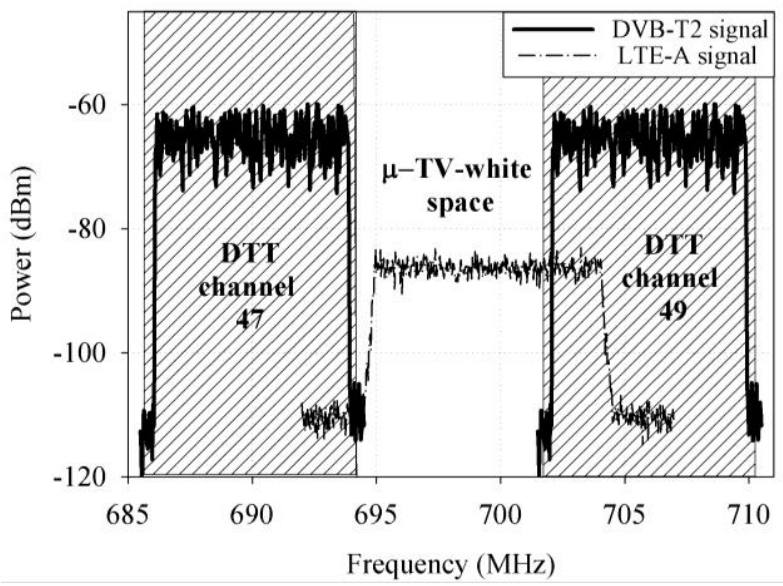

Fig 3. Case B: LTE-A Femtocell (10 MHz bandwidth) transmitted in a $\mu$ TVWS located between two DVB-T2 channels.

\section{MethodOLOGY}

The results have been obtained by laboratory emulation and validated through field measurements using professional equipment. To determine the maximum allowable EIRP for LTE-A femtocell we carried out the following four steps:

1. Measure the protection ratios between DVB-T2 and LTE-A (Downlink) systems. The Protection Ratio is defined as the minimum required value of the difference between the useful (DVB-T2) and the interfering (LTE-A) signal levels, expressed in $\mathrm{dB}$, at the receiver input to accomplish with a specific quality requirement.

2. Determine the maximum allowable interference power of the femtocell at the DTT receiver $\left(P_{i}^{L T E-A}\right)$, for the considered scenarios, using the protection ratios obtained in the previous step:

$$
P_{i}^{L T E-A}=P_{\min }^{D V B}-P R
$$

where $P R$ is the protection ratio obtained in the previous step and $P_{\min }^{D V B}$ is the sensitivity (minimum required received power) of the DVB-T2 receiver. This parameter depends on the DVB transmission mode used.

3. Calculate the path loss between an indoor LTE-A femtocell and the DVB-T2 receiving antenna $\left(P L_{i 20}\right)$, this parameter depends on the scenarios under study and can be computed as:

$$
P L_{i 2 o}=F S L+\sum_{i=1}^{N} n_{i} * l_{i}-G_{r}
$$

where $F S L$ is the free space loss, which is calculated using the propagation model UIT-R 525-2 [16], $n_{i}$ is the number of obstacles that are crossed by the direct path between the femtocell and the DVB-T2 antenna, and $l_{i}$ is the attenuation caused by the obstacle. 
This parameter depends on the type of the obstacle (wall, ceiling, windows, floor, and furniture) and the frequency. Typical attenuation values of these kind of obstacles can be found in [17]. $G_{r}$ is the receiving antenna gain. This parameter is based on the antenna directivity and the angular antenna discrimination, which is associated with the vertical radiation pattern of the DTT antenna.

4. The maximum EIRP that could transmit an indoor LTE-A femtocell $\left(E I R P_{\max }^{L T E-A}\right)$, can be obtained such as:

$$
E I R P_{\max }^{L T E-A}=P_{i}^{L T E-A}+P L_{i 2 o}
$$

where $P_{i}{ }^{L T E-A}$ is the interfering allowable power of the femtocell and $P L_{i 2 o}$ is the indoor to outdoor path loss, which can be considered as a gain to determine the maximum EIRP for a LTE-A femtocell.

\section{A. Protections Ratio Measurements Setup}

The measurement of protection ratios have been carried out in laboratory conditions using the subjective failure point method specified in the Recommendation ITU-BT 2215 [18], as shown in Fig. 4. The DTT power was set to $60 \mathrm{dBm}$ and the interfering signal (LTE-A) was increased in $0.1 \mathrm{~dB}$ steps, waiting 20 seconds in each step to detect possible errors in a real video. If an error is detected, the previous interfering power is taken as the maximum allowed [19]. As a function of DVB-T2 and LTE-A carrier separation, the protection ratios are measured by varying the central frequency of the LTE-A signal from co-channel to adjacent channel (case A) and between two DVB-T2 channels (case B ) in steps of $0.5 \mathrm{MHz}$.

For DVB-T2, a signal generator with channel emulator (R\&S SMU 200) has been used. A Rice channel model for DVB-T2 has been considered, which is equivalent to assume line-of-sight between the DTT transmitters to the rooftop receiver (DVB-T2 link). The LTE-A signal has been generated using a second signal generator (Aeroflex SGD-6).

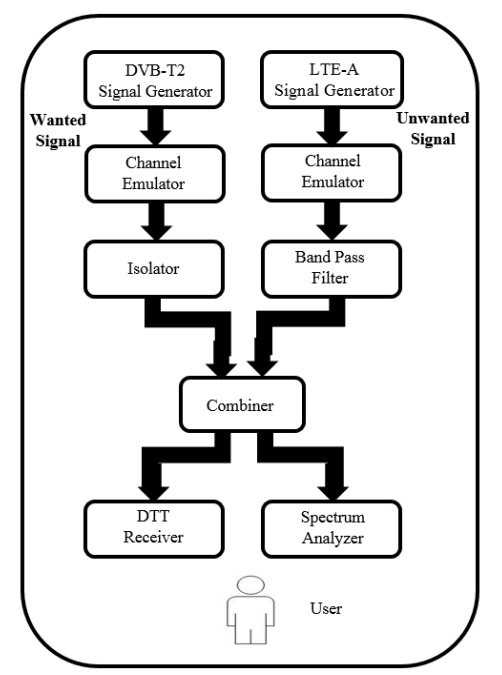

Fig 4. Experimental laboratory set-up to measure interference protection ratios between DTT and LTE-A signals.
TABLE. I

DTT AND LTE-A REFERENCE SIGNAL PARAMETERS

\begin{tabular}{cc}
\hline \hline & DTT \\
\hline \hline Parameter & Fixed Rx \\
\hline Standard & DVB-T2 \\
Modulation & $256-$ QAM \\
Code Rate & $2 / 3$ \\
FFT & 32 K Extended \\
Guard Interval & $1 / 128(28 \mu \mathrm{s})$ \\
Pilot Pattern & PP7 \\
CNR (RICE) & $20 \mathrm{~dB}$ \\
Bandwidth & $8 \mathrm{MHz}$ \\
\hline \hline & \\
\hline \hline Parameter & Downlink Value \\
\hline Standard & LTE-A \\
Multiplex & OFDM \\
Duplexing & FDD \\
Modulation & QPSK \\
FFT & 512,1024 \\
Guard Interval & Normal \\
Traffic loading & $(4.7 \mu$ s first symbol, 5.2 $\mu$ s next $)$ \\
Bandwidth & IDLE / 100\% \\
& $5 / 10$ MHz \\
\hline
\end{tabular}

Table I presents the configuration parameters employed for DVB-T2 and LTE-A. The DVB-T2 mode is the one used in the United Kingdom broadcast networks. We have assumed a bandwidth of $8 \mathrm{MHz}$ for the DVB-T2 signal (Europe). For the LTE-A-DL signal (Downlink from femtocell to LTE-A users) we considered different bandwidths and traffic loads, in order to evaluate the worst case in the scenarios under analysis. We have set a bandwidth of 5 and $10 \mathrm{MHz}$ and different traffic loads (idle and fully load). A set of 5 different DTT receivers, including TV screens and set top boxes, were used to evaluate the quality of video reception. The modulation does not change significantly the shape of the LTE-A signal spectrum and therefore does not influence the results. For this case we assumed QPSK, the same that is chosen in ITU studies [19].

\section{B. Field Trials Setup}

The field trials configuration is shown in Fig. 5. They were carried out at the Universitat Politècnica de Valencia (UPV), Spain. The DTT antenna was located about $500 \mathrm{~m}$ far from the DVB-T2 transmitter. A LTE-A femtocell was placed at different indoor locations.

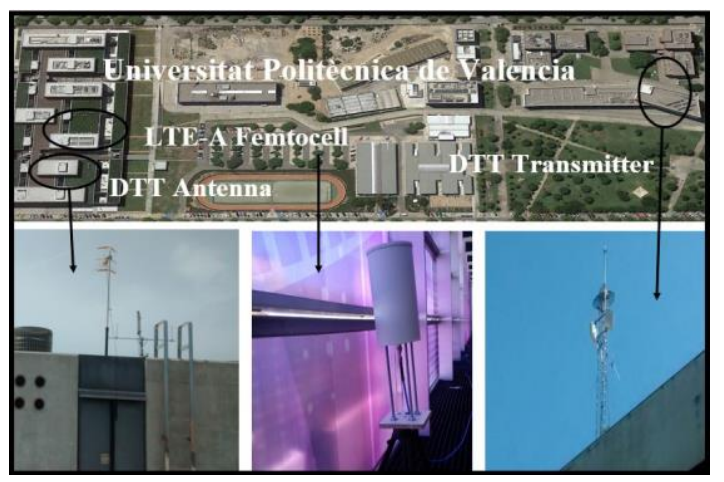

Fig 5. Location of the scenarios under analysis in field measurements. 
The transmitter used for generating the DVB-T2 signal is a Multi-standard modulator DekTec DTA-115 Multi-Standard VHF/UHF. The transmitter output was connected to amplification and supply stages and distributed to two sector panel antennas. These antennas have $11 \mathrm{dBi}$ gain each and their radiation patterns have $28^{\circ}$ and $62^{\circ}$ beamwidths in the horizontal and vertical planes respectively.

The LTE-A signals of the femtocell have been generated with the same equipment used in the laboratory. The LTE-A femtocell was connected to an omnidirectional antenna. This antenna has $2 \mathrm{dBi}$ gain in the DTT band. Finally, the DTT receiving antenna was connected on one side to a DTT receiver and on the other side to a Spectrum Analyzer (Anritsu Spectrum Master MS2720T). This spectrum analyser is employed to provide power spectrum measurements.

The field measurements have been performed taking into account the next steps: first of all, the quality of the video reception for DVB-T2 in the absence of the LTE-A signal emission is measured. Secondly, the received signal power from the LTE-A femtocell in the absence of DVB-T2 signal is measured. Thirdly, the indoor to outdoor path loss between the LTE-A femtocell and the DTT antenna is measured. Fourthly, the LTE-A femtocell was configured to emit with different parameters of bandwidth, frequency, power and traffic loads. Finally, the DVB-T2 and LTE-A signals are transmitted simultaneously to measure the protection ratios and the maximum EIRP of the LTE-A femtocell.

\section{RESULTS}

\section{A. Laboratory Measurements \\ 1) Protection Ratio Measurements}

The protection ratios are computed as a function of the separation between the DVB-T2 and the LTE-A central carriers, varying from co-channel to non-overlapping in steps of $0.5 \mathrm{MHz}$. In addition, the overlapping that suffers the DVB$\mathrm{T} 2$ channel is also presented. The overlapping is defined as the quantity of undesired spectrum (LTE-A signal) that interferes the useful spectrum (DVB-T2 signal), and it is calculated as the percentage of bandwidth of the interfering spectrum that is over the total bandwidth of useful spectrum.

\section{a) Effect of LTE-A traffic load}

Fig. 6 shows the protection ratios for a DVB-T2 useful signal interfered by a $5 \mathrm{MHz}$ LTE-A signal, for two different traffic loads (100\% and Idle).

As can be observed, the protection ratios are more restrictive for the LTE-A signal at $100 \%$ load, as could be expected for this OFDM system [5]. Concerning on the carrier separation, the protection ratios are very restrictive for cochannel, since all the LTE-A spectrum falls into the DVB-T2 channel, but have a significant improvement when the LTE central carrier is between 1 and $3 \mathrm{MHz}$ (Overlapping between 0 and $20 \%$ ) regarding to the DVB-T2 channel edge, meaning that some overlap, or at least no guard band, could be set under certain circumstances.

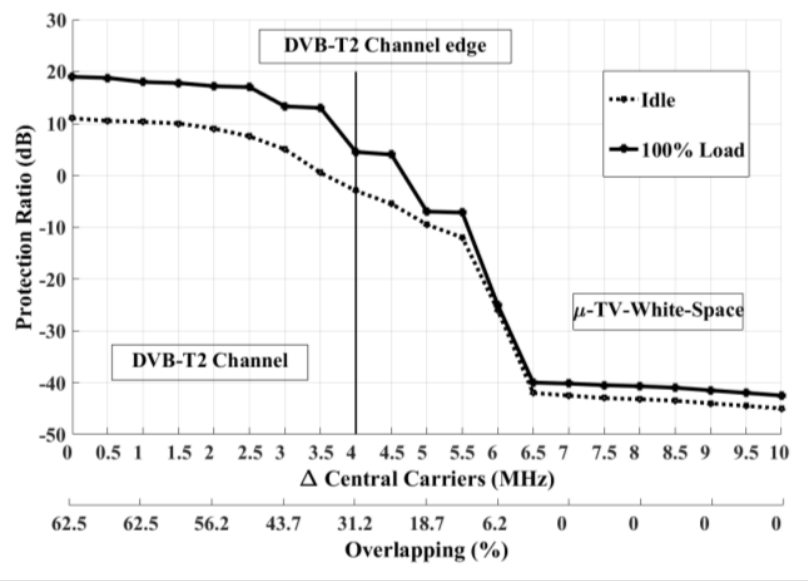

Fig 6. Protection ratios for fixed DTT reception, interfered by LTE-A-DL with a bandwidth of $5 \mathrm{MHz}$, for different traffic loads.

b) Effect of the LTE-A bandwidth

Case A: Co-channel and adjacency channel

Fig. 7 illustrates the protection ratio measurements for the same DVB-T2 signal interfered by a fully loaded LTE-A DL channel (worst case), for two different bandwidths: $5 \mathrm{MHz}$ and $10 \mathrm{MHz}$.

As in the previous case, the measurements are performed from co-channel to first adjacent channel (no spectrum overlap between the two systems). Results show how much the protection ratios for LTE $10 \mathrm{MHz}$ are more restrictive to those for $5 \mathrm{MHz}$, due to the different out-of-band fall for each LTE-A channelization, and mainly due to the difference in the overlapped bandwidth with respect to a DVB-T2 channel [5].

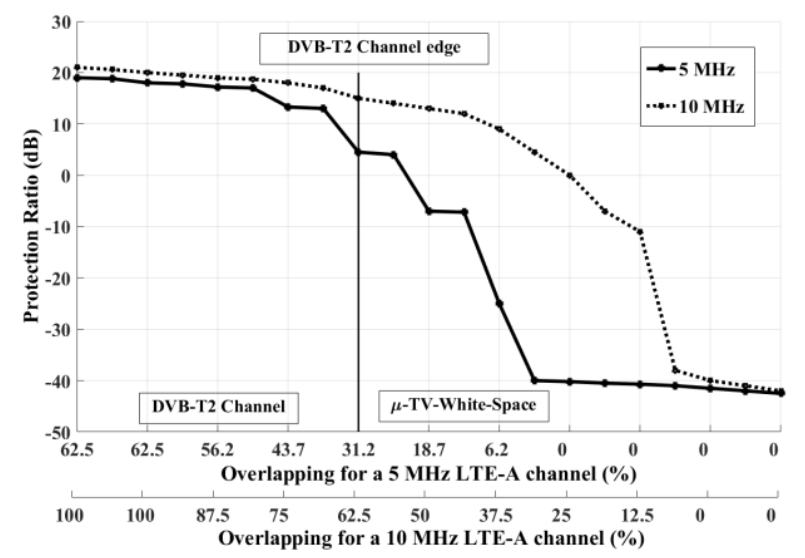

Fig 7. Protection ratios for fixed DTT reception, interfered by LTE-A-DL, for different LTE-A bandwidth.

\section{(2) Case B: Double Adjacency}

Fig. 8 shows the protection ratio measurements for a fully load LTE-A signal interfering between two DVB-T2 channels, for two different bandwidths: $5 \mathrm{MHz}$ and $10 \mathrm{MHz}$. Also, the overlapping that suffers the two DVB-T2 channels with respect to the LTE-A central carrier is represented. 


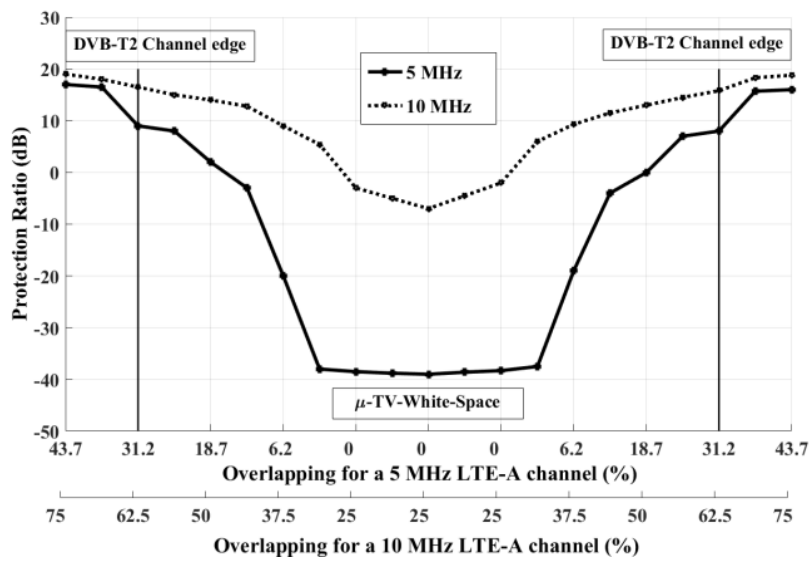

Fig 8. Protection ratios for two DVB-T2 channels, interfered by LTE-A-DL, for different LTE-A bandwidth.

In this case, protection ratios are also more restrictive for the $10 \mathrm{MHz}$ since LTE-A channel uses a $\mu$ TVWS with lower bandwidth ( $\mu$ TVWS of $8 \mathrm{MHz}$ bandwidth). Thus, the LTE signal is always overlapped between the two DVB-T2 channels. On the contrary, the $5 \mathrm{MHz}$ LTE-A channel has up to $1.5 \mathrm{MHz}$ of guard band with respect to the two DVB-T2 useful channels. Hence, the results of case study B are more interfering than those from case study A.

\section{B. Field Trials}

The three scenarios proposed in Section II have been evaluated by field measurements and were compared with laboratory measurements. Before the measurement process, it is necessary to find the location of the LTE-A femtocell that determined the lowest path loss from the femtocell to the DTT receiving antenna (work cases). The femtocell was always placed at 90 centimetres of height above the ground of the top floor.

Protection ratios, path losses and received power were measured employing a spectrum analyser at the receiver input, so both the directivity and the angular discrimination of the vertical radiation pattern of the DTT antenna are included in the measurements. The power levels for each frequency bin are obtained through the average detection principle.

\section{1) Path loss}

Taking into account a null angular discrimination antenna (worst case), path loss from the femtocell to the DTT receiving antenna were measured. Table II shows the indoor to outdoor path losses obtained for each of the three scenarios.

TABLE. II.

PATH LOSS BETWEEN FEMTOCELL AND DTT RECEIVING ANTENNA FOR THE SCENARIOS.

\begin{tabular}{cccc}
\hline \hline & Scenario 1 & Scenario 2 & Scenario 3 \\
\hline \hline $\begin{array}{c}\text { Path Loss } \\
(\mathrm{dB})\end{array}$ & 62 & 54 & 47 \\
\hline
\end{tabular}

The most restrictive path loss is $47 \mathrm{~dB}$ in the scenario 3 (worst case), in this case, both the femtocell and the receiving antenna are located at the same height, with line-of-sight between the antennas and glass in the middle of the path. In scenario 2 results are very similar, we can observe $7 \mathrm{~dB}$ extra losses compared to scenario 3 , because the larger path length of the interfering signal. This implies that the femtocell of the scenario 2 could transmit up to $7 \mathrm{dBm}$ more than in the worst scenario (scenario 3). Scenario 1 is the less critical mainly because of there is no line-of sight between the antennas and the kind of obstacle that LTE-A signal has to go through.

The results are compared with a campaign of measurements proposed by British Broadcasting Corporation (BBC) [20]. The BBC evaluated the path loss between White Space device and DTT antenna, considering the same three scenarios. However, there are some differences to keep in mind between the measurements performed in our work and those driven by BBC. While our study was carried out in an area with high density of buildings, their measurements were performed in a private house of a region with lower density of constructions. In addition, the distance between the antennas (DTT and LTE femtocell) as well as the height between them are slightly different between both studies. In our case, path loss is between 2 and $4 \mathrm{~dB}$ higher than those provided by BBC, being the scenario 2 which presents greater difference. Also, it should be pointed out that our results are a bit different due the slightly differences in the scenarios setup, and other factors that affect the path loss, such as the environment type, weather, etc. However, the results are consistent with those presented by BBC.

\section{2) Maximum allowable EIRP for the indoor LTE-A Femtocell}

The useful DTT received power was set to the minimum required received power, $P_{\min }^{D V B}$, for $95 \%$ of location probability for the DVB-T2 reference mode $(-73 \mathrm{dBm})$ [21]. This represent the worst case (i.e. with the DTT receiver antenna located at the edge coverage of DVB-T2 transmitter). Then, considering the protection ratio as well as the path loss obtained above, the maximum femtocell EIRP allowed was measured.

\section{a) Case A: Co-channel and adjacency channel.}

LTE-A $5 \mathrm{MHz}$ bandwidth

Fig. 9 shows the maximum EIRP that could transmit the indoor LTE-A femtocell for the three proposed scenarios obtained in field trials, for the fully load traffic (worst case) and in terms of the overlapping from the LTE channel to the DTT channel. Furthermore, the maximum femtocell EIRP allowed in the scenario 3 (worst case) is compared with that obtained in laboratory. Maximum EIRP in the scenario 3 is between 0.3 and $4 \mathrm{~dB}$ more restrictive that laboratory measurements, due mainly to the channel fading, which affects the signals in a real transmission scenario. 


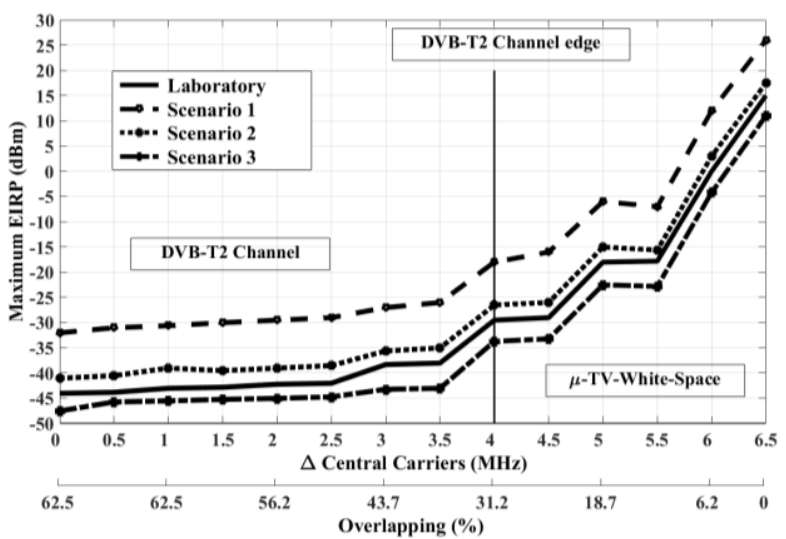

Fig 9. Comparison between laboratory and field measurements, for a $5 \mathrm{MHz}$ LTE-A channel in the case A.

In the other two scenarios the difference between field trials and laboratory measurements was similar or smaller that for the scenario 3 . it should be noted that results indicate that it is not feasible to allocate the LTE-A carrier within the DVB-T2 channel, due to the low power (between -46 and $-34 \mathrm{dBm}$, scenario 3 ) and (between -31 and $-19 \mathrm{dBm}$, scenario 1) that could transmit the femtocell without interfering the DVB-T2 signal. Thus, the co-channel approach can be disregarded. EIRP typical value that could be transmit an indoor LTE Small cell is $21 \mathrm{dBm}$ in licenced bands [22].

The maximum EIRP that could transmit the femtocell in the adjacent channel without band guard (overlapping 0\%) is 11 $\mathrm{dBm}$ in the most restrictive case (scenario 3 ). In the case of the scenario 1 and 2 the maximum allowed EIRP is $27 \mathrm{dBm}$ and $18 \mathrm{dBm}$, respectively.

Moreover, if the LTE-A signal transmit with about $20 \%$ overlapping over the DVB-T2 channel, it is possible to transmit up to $-22 \mathrm{dBm}$ (scenario 3 ) or $-6 \mathrm{dBm}$ (scenario 1) between the channels of both technologies. Therefore, overlapping $20 \%$ into the DTT signal, the femtocell could transmit, but with a PIRE reduction of $33 \mathrm{~dB}$ compared to transmit in adjacent channel without guard band.

\section{(2) LTE-A $10 \mathrm{~Hz}$ bandwidth}

In Fig. 10 could be observe similar results for a $10 \mathrm{MHz}$ LTE-A channel compared to those obtained for $5 \mathrm{MHz}$ bandwidth. Like in the $5 \mathrm{MHz}$ case, a co-channel transmission between a LTE femtocell and a DVB-T2 signal is not feasible because of the low PIRE allowed for the femtocell in all scenarios.

For adjacent channel transmissions, the femtocell could transmit up to $11 \mathrm{dBm}$ (scenario3) and $26 \mathrm{dBm}$ (scenario 1) with an offset of $9 \mathrm{MHz}$ (without guard band) between DVBT2 and LTE-A central carriers. In this case, for an overlapping of $20 \%$ the femtocell could transmit up to $-21 \mathrm{dBm}$ and $-5 \mathrm{dBm}$ for the scenario 3 and 1 , respectively. It should be noted that for the same overlapping the allowed EIRP of the femtocell does not depend on the LTE-A signal bandwidth.

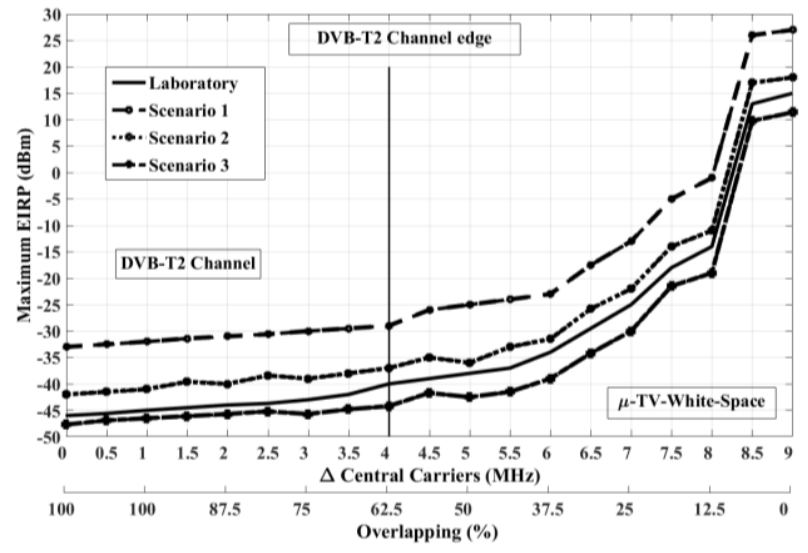

Fig 10. Comparison between laboratory and field measurements, for a 10 MHz LTE-A channel in the case A.

b) Case B: Double Adjacency.

(1) LTE-A $5 \mathrm{MHz}$ bandwidth

Fig. 11 shows that the maximum allowable EIRP that a femtocell could transmit is $11 \mathrm{dBm}$ for the most restrictive case (scenario 3) and $27 \mathrm{dBm}$ for the best case (Scenario 1). Thus, results demonstrate that transmitting a $5 \mathrm{MHz}$ LTE-A channel using a micro-TV-White-Space between two DVB-T2 channels is feasible.

EIRP typical value is $21 \mathrm{dBm}$ in the $3400 \mathrm{MHz}$ band for an indoor LTE-Small Cell, it could be find in the recommendations [22][23]. The same EIRP was considered by Small Cell Forum in the $1900 \mathrm{MHz}$ and $2600 \mathrm{MHz}$ bands [24]. Comparing this value with those obtained in this paper, for the worst case (scenario 3) the maximum allowed EIRP is $10 \mathrm{dBm}$ lower. However, the DTT band offers great propagation conditions. The difference in path loss are between 10 to $15 \mathrm{~dB}$ compared to the $1900 \mathrm{MHz}$ and 3400 $\mathrm{MHz}$ band. Therefore, the lower path losses could balance those $10 \mathrm{~dB}$ degradation in the EIRP.

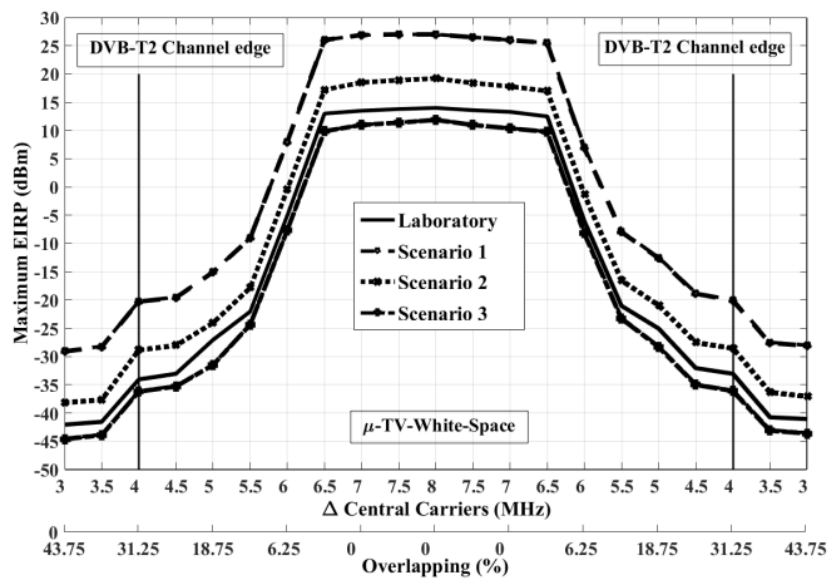

Fig 11. Comparison between laboratory and field measurements, for a $5 \mathrm{MHz}$ LTE-A channel in the case B. 
(2) LTE-A $10 \mathrm{~Hz}$ bandwidth

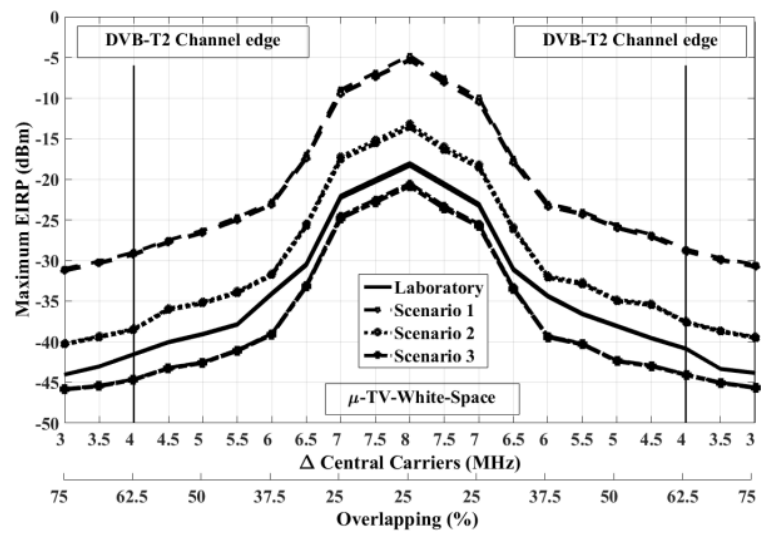

Fig 12. Comparison between laboratory and field measurements, for a 10 MHz LTE-A channel in the case B.

Fig. 12 shows the maximum EIRP that could transmit a femtocell when its central carrier is between two DVB-T2 channels for the proposed scenarios. One can observe that a femtocell using a $\mu$ TVWS of $8 \mathrm{MHz}$ between two DVB-T2 channels, could transmit a $10 \mathrm{MHz}$ LTE-A signal when the power is limited to $-19 \mathrm{dBm}$ (scenario 3) or $-7 \mathrm{dBm}$ (Scenario 1) without causing interference to the roof-top DVB-T2 receiver. Therefore, this study case is not feasible due to the very constrained power conditions of the femtocell. Nevertheless, this case could be possible if the LTE-A signal is between two consecutive no occupied DTT channels.

\section{3) Capacity Analysis}

To estimate how much capacity improvement a sharing spectrum femtocell could provide to an existing network, we compare it with the current small cells operating in licensed bands. While the typical EIRP values allocated for the standard femtocells have an average of $21 \mathrm{dBm}$, the maximum power that an indoor LTE-A femtocell in a sharing scenario could transmit is $11 \mathrm{dBm}, 18 \mathrm{dBm}$ and $27 \mathrm{dBm}$ in the scenarios 3, 2 and 1, respectively, as described in section IV. For the worst case (scenario 3), there is a power reduction of $10 \mathrm{~dB}$ compared to the licensed band femtocells, which obviously will provide a capacity below the maximum boundary.

Fig. 13 compares the maximum capacity available in coherent systems for different configurations: SISO, $2 \times 2$ MIMO and $4 \times 4$ MIMO, to the capacity that could be offered with a sharing-spectrum femtocell that is constrained to EIRP of $10 \mathrm{~dB}$ below the standard cells. The capacity has been calculated for Gaussian MIMO channels, with the Shannon capacity for SISO channels as reference [25]. As can be seen in Fig. 13, the capacity of a sharing-spectrum femtocell with $10 \mathrm{~dB}$ power reduction is in the order of $50 \%$ of the maximum, meaning that the use of such white spaces or channels adjacent to DTT transmissions, provide still a significant contribution to the total Network capacity.

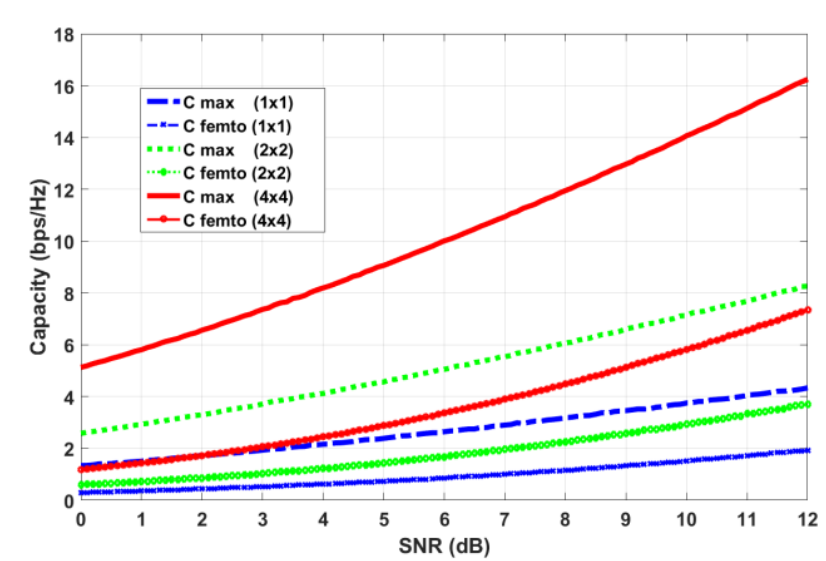

Fig 13. Capacity of the LTE-A femtocell respect to the theoretical maximum capacity.

In a study carried out by the Small Cell Forum, the peak rate for indoor scenarios through simulations was analysed. In this study a spectral efficiency of $6 \mathrm{bps} / \mathrm{Hz}$ for the downlink with MIMO $2 \times 2$ configuration was obtained [24]. The recommendation ITU-R 2134 define a peak spectral efficiency of $15 \mathrm{bps} / \mathrm{Hz}$ for LTE advance downlink with MIMO $4 \mathrm{x} 4$ configurations [26]. These results are in line with the maximum capacity shown in Fig. 13, where the capacity values obtained for high SNRs with a power reduction of 10 $\mathrm{dB}$ are $2 \mathrm{bps} / \mathrm{Hz}, 4 \mathrm{bps} / \mathrm{Hz}$ and $8 \mathrm{bps} / \mathrm{Hz}$, approximately. Therefore, the downlink throughput that could be obtained for high SNRs with such power reduction could reach $36 \mathrm{Mbps}$ for a $5 \mathrm{MHz}$ channel with $4 \times 4 \mathrm{MIMO}$ configuration.

It is worth noting that in this paper we have measured the LTE Downlink carrier as the interferer to the DTT receiver, which is valid for the FDD mode, but also for carrier aggregation case to reinforce the downlink capacity.

\section{CONCLUSIONS}

The use of white spaces in the DTT band for spectrum sharing between Mobile and Broadcast technologies is currently one of the hot topics to satisfy the spectrum demand required by the IMT-Advanced technologies, and it is also an efficient alternative to manage radio spectrum.

In this paper we have demonstrated, based on measurements in real scenarios, the feasibility of using TV-White-Spaces in the DTT band for spectrum sharing between indoor LTE-A femtocells and DVB-T2 networks with fixed rooftop reception. The paper specifically analyses the case of indoor LTE-A femtocells in coexistence with DVB-T2 transmissions, from the allocation of adjacent frequencies in the same band (white spaces) to the $100 \%$ overlapping case (co-channel). This scenario has been called micro-TV-White-Space ( $\mu$ TVWS), in the sense that takes advantage of small areas where the DVB-T2 signal is weak and not used, mainly indoors. Therefore, this proposal can be considered as a possible solution to satisfy some of the spectrum demand required by the IMT-Advanced technologies, being spectrum 
sharing an efficient option for allocation, upon the conditions and parameters established with this analysis.

The results provided are based on the comparison between emulated and measured scenarios. The emulation of the scenarios in laboratory is supported by the generation of synthetic signals, hardware emulation of radio channels and use of commercial receivers to evaluate the quality of the decoded signals and so the limits of mutual interference. These emulations have been afterwards validated through a large number of field measurements using professional equipment. All results correspond to the most restrictive cases, which considers a full load in the LTE-A in Downlink, minimum DTT required received power, and the lowest propagation losses between the femtocell to the receiving antenna for the proposed scenarios. Three different scenarios were analysed keeping these restrictions.

Protection ratios obtained through field measurements are within a range from 0.3 to $4 \mathrm{~dB}$ greater than those measured under laboratory conditions, probably caused by additional signal fading inherent to the path conditions. Therefore, the maximum allowable EIRP for the coexisting LTE-A femtocell can be calculated from the protection ratios obtained in laboratory plus a correction factor, channel dependent, which could be obtained by channel estimation. Such functionality can be implemented in cognitive femtocells or, in the worst case, an additional protection ratio of $4 \mathrm{~dB}$ could be used.

The main outcome of this study case is finding the appropriate EIRP restrictions for a LTE-A femtocell to operate in frequencies adjacent, or even partially overlapped, to a DVB-T2 channel.

Our results indicate that is feasible to allocate LTE-A carriers in adjacency to a DVB-T2 channel under certain constrains. The results fix the power limit for an indoor LTEA femtocell in $+11 \mathrm{dBm},+18 \mathrm{dBm}$ and $+27 \mathrm{dBm}$, for the analysed scenarios 3,2 and 1, respectively. These values apply to a $5 \mathrm{MHz}$ and $10 \mathrm{MHz}$ bandwidth LTE-A signals for adjacent channel without guard band. In the most restrictive case (scenario 3) there is a power reduction of $10 \mathrm{~dB}$ compared to the EIRP values of a typical indoor LTE small cell in licensed bands. The impact of this power limitation on the achievable capacity could be approximately the $50 \%$ of the maximum capacity of current LTE-A MIMO cells, reaching values of about $8 \mathrm{bps} / \mathrm{Hz}$ and downlink throughputs of $36 \mathrm{Mbps}$ in MIMO $4 \times 4$ configurations and $5 \mathrm{MHz}$ bandwidth.

Moreover, if the LTE-A carrier is allocated with about $20 \%$ overlapping over the DVB-T2 channel, the femtocell still could transmit with an EIRP reduction of $33 \mathrm{~dB}$. The cochannel case is disregarded in any scenario, as from the results obtained it would restrict the femtocells EIRP to $-46 \mathrm{dBm}$, which would lead to very scarce coverage or extremely low efficiency.

The case of an indoor LTE-A femtocell using a white space between two DVB-T2 adjacent channels is possible for a 5 MHz LTE signal, with the same results presented for adjacent channel without band guard. For the LTE-A $10 \mathrm{MHz}$ bandwidth is not feasible due to the very constrained power conditions of the femtocell (EIRP restricted to $-19 \mathrm{dBm}$ ).
Nevertheless, this case could be possible if the LTE-A signal is between two consecutive non occupied DTT channels.

The analysis and measurements performed in this paper are for LTE Downlink carrier as interferer to a DTT receiver, which are valid for the FDD mode, but also for carrier aggregation (LTE-A CA) to reinforce the downlink capacity.

\section{ACKNOWLEDGMENTS}

Authors would like to thank Dr David Gómez-Barquero for his fruitful discussions and comments to this work. This work was supported by the Ministerio de Educación y Ciencia, Spain ("DEFINE5G" TEC2014-60258-C2-1-R and "ARCO5G" TEC2014-56469-REDT), by the European FEDER funds.

\section{REFERENCES}

[1] Presentations by ABI Research, Picochip, Airvana, IP access, Gartner, Telefonica España, 2nd Intl. Conf. Home Access Points and Femtocells; available online at http://www.avrenevents.com/.

[2] Cisco Visual Networking Index: Global Mobile Data Traffic Forecast Update 2015-2020 White Paper, Feb. 1, 2016, available at http://www.cisco.com/c/en/us/solutions/collateral/serviceprovider/visual-networking-index-vni/white_paper_c11-520862.html/.

[3] ITU-R, "Future spectrum requirements estimate for terrestrial IMT". Report ITU-R M.2290. December 2013.

[4] ITU-R, "Final Acts World Radiocommunication Conference (WRC12), "November 2012.

[5] J. Ribadeneira, G. Martinez, D. Gomez, N. Cardona, "Interference Analysis Between Digital Terrestrial Television (DTT) and 4G LTE Mobile Networks in the Digital Dividend Bands," IEEE Transactions on Broadcasting ,vol. 62, no. 1, pp. 24-34, March 2016.

[6] G. Baruffa, M. Femminella, F. Mariani, and G. Reali, "Protection Ratio and Antenna Separation for DVB-T/LTE Coexistence Issues," IEEE Communications Letters, vol.17, no.8, pp.1588-1591, August 2013.

[7] ITU-R, "Planning Criteria, Including Protection Ratios, for Second Generation of Digital Terrestrial Television Broadcasting Systems in the VHF/UHF Bands," Recommendation ITU-R BT.2033, January 2013.

[8] DVB SB2122, "Study on Specification and Use of in-line Filters to Reduce Interference in Broadcast Bands from Mobile Base Stations," DVB White Paper, March 2014.

[9] M. Fuentes, C. Garcia, E. Garro, D. Gomez, and N. Cardona, "Coexistence of Digital Terrestrial Television AND Next Generation Cellular Networks in THE $700 \mathrm{MHz}$ Band." IEEE Wireless Communications. December 2014.

[10] M. Fuentes, E. Garro, C. Garcia, D. Gomez, N. Cardona, "Coexistence of LTE and DTT services in the First and Second Digital Dividends. Planning studies and potential approaches." IC1004 TD (14)10034, May 2014.

[11] L. Polak, et al., "Study of coexistence between indoor LTE femtocell and outdoor-to-indoor DVB-T2-Lite reception in a shared frequency band," EURASIP Journal on Wireless Communications and Networking, vol. 2015, no. 114, April 2015.

[12] Pascal Lamy, "Results of the work of the high level group on the future use of the UHF band (470-790 MHz), September, 2013.

[13] CEPT ECC Report 224,'Long Term Vision for the UHF broadcasting band. On long term vision for the UHF broadcasting band out for public consultation," November, 2014.

[14] ITU-R, "Provisional Final Acts World Radiocommunication Conference (WRC-15)," November 2015.

[15] J. Beek, J. Riihijärvi, A. Achtzehn, P. Mähönen, "UHF white space in Europe - a quantitative study into the potential of the $470-790 \mathrm{MHz}$ band". IEEE International Symposium on Dynamic Spectrum Access Networks, 2011.

[16] ITU-R P.525-2, “Calculation of free-space attenuation”, 1994.

[17] Digi, "Indoor Path Loss", June, 2012.

[18] ITU-R, "Measurements of protection ratios and overload thresholds for broadcast TV receivers". Recommendation ITU-R BT.2215. June 2012. 
[19] ITU-R, "Planning criteria, including protection ratios, for second generation of digital terrestrial television broadcasting systems in the VHF/UHF bands". Recommendation ITU-R BT.2033. January 2013.

[20] BBC Arquiva, "White Paper 288: WSD Coexistence Testing at the Building Research Establishment: An Experimental Validation of Ofcom Regulatory Proposals", December 2014.

[21] EBU TECH 3348, "Frequency and Network Planning Aspects of DVBT2", Report, 2014.

[22] ITU-R M.2135-1, "Guidelines for evaluation of radio interface technologies for IMT-Advance", 2010.

[23] ETSI TR 136 932, "LTE; Scenarios and requirements for small cell enhancements for E-UTRA and E-UTRAN", October 2014.

[24] Small Cell Forum, "Small cell and Wi-Fi coverage study", February 2014

[25] Lizhong Zheng, etc " Communication on the Grassmann Manifold: A Geometric Approach to the Noncoherent Multiple-Antenna Channel, IEEE Transactions on Information Theory, vol. 48, no. 2, February 2002

[26] ITU-R M.2134, "Requirements related to technical performance for IMT-Advanced radio interface(s)", 2008.

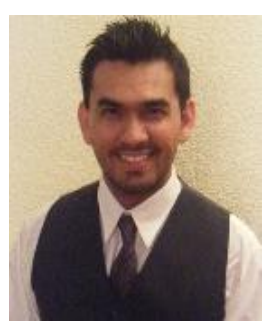

Gerardo Martínez Pinzón received a double Telecommunications Engineer degree at Technical University of Valencia, Spain and Saint Thomas Aquinas University, Colombia. In 2013, he has finished a Master in Development of Mobile Communications Systems. More than 3 years of experience in the mobile communications, digital terrestrial television and electronic security systems fields. He has developed research activities and consulting providing recommendations on multiple projects from the initial design stages to planning, optimization, coexistence and deployment. Specialized in the latest telecommunications technologies like 4G-LTE (Long Term Evolution) mobile communications or DVB-T, DVB-T2 and ISDB-T digital terrestrial television (DTT) standards. His current research activities are focused on efficient spectrum management through the use of cognitive radio technologies and radio access technologies for Internet of Things.

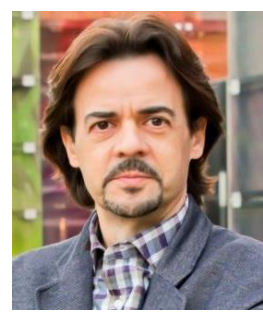

Prof. Narcís Cardona received the M.Sc. degree in Communications Engineering from the ETSI Telecommunications at the Polytechnic University of Catalunya in 1990, and the Ph.D. in Telecommunications from the Polytechnic University of Valencia in 1995. Since October 1990, he is with the Communications Department of the Polytechnic University of Valencia (UPVLC). Prof Cardona is in head of the Mobile Communications Group at the UPVLC, with 30 researchers including assistant professors \& research fellows. Additionally he is the Director of the Mobile Communications Master Degree (since 2006) and Vice-Director of the Research Institute of Telecommunications and Multimedia Applications (iTEAM) since 2004. Prof Cardona has led and participated to National research projects and to European projects, Networks of Excellence and other research forums, always in Mobile
Communications aspects. At European scale, he has been Vice-Chairman of COST273 Action, Chair of the WG3 of COST2100 in the area of Radio Access Networks, and he is currently the Chairman of the EU Action COST IC1004 since May 2011. From his research work, Prof Cardona has authored eight patents, several books and above 160 research papers. His current areas of interest include mobile channel characterization; planning and optimization tools for cellular systems, RRM techniques applied to personal communications and broadcast cellular hybrid networks.

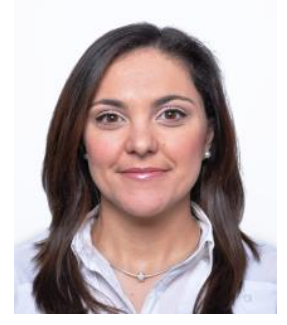

Concepción Garcia-Pardo attended the Universidad Politécnica de Cartagena, where she graduated in Telecommunication Engineering in 2007 and she received the M.Sc. degree in Information Technologies and Communications in 2008. In 2007 she started her Ph.D. studies focused on the experimental characterization of the path channel for systems with large bandwidth and multiple antennas. Between 2008 and 2010 she stayed two times as a guest researcher at the Telecommunications and Electromagnetic Compatibility group (TELICE) at the Lille 1 University in France. In 2012 she received her $\mathrm{PhD}$ degree with European mention and qualification cum laude, from the Universidad Politécnica de Cartagena, and $\mathrm{PhD}$ degree in Microwaves and Microtechnologies with qualification Très Honorable from the Lille 1 University. Her PhD Thesis was awarded the special prize from the Universidad Politécnica de Cartagena in 2013. She is the author of several publications in many national and international journals and conferences. In 2011 and 2012 she was a lecturer at the Universidad Politécnica de Cartagena, in the area of Signal Theory and Communications. In 2012 she joined the Mobile Communications Group as a researcher, where she has participated in some projects related to broadcasting and mobile communications. Her current work is focused on the study of interferences between television systems and other mobile communication systems, radio channel characterization in special and confined environments and body area networks.

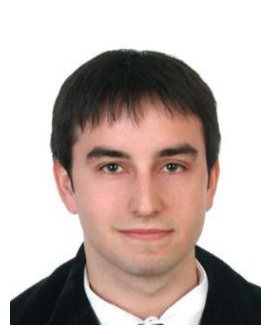

Alejandro Fornés-Leal received the telecommunications engineering degree at Universitat Politècnica de València, València, Spain, in 2014. Since February 2014, he is with the Mobile Communication Group, Institute of Telecommunications and Multimedia Applications (iTEAM), València, as a researcher. During this first year he participated in some projects related to ultrawideband characterization in emergencies and confined environments, and Body Area Networks. His current work is focused on the development of medical applications and the characterization of the ultrawideband channel for body area networks. 


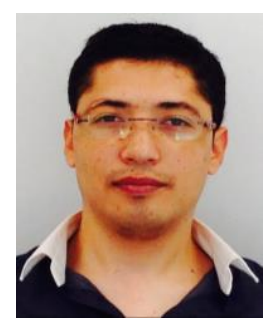

Jefferson Ribadeneira-Ramírez he received the Electronic and Computation Engineering degree in 2008 from the Escuela Superior Politécnica de Chimborazo (ESPOCH), Riobamba, Ecuador. In 2010, he was recipient of a scholarship for studies of excellence, granted by the SENESCYT (Secretaría Nacional de Educación Superior, Ciencia, Tecnología e Innovación) of Ecuador. In 2013, he obtained the M. Sc. degree in Technologies Systems and Telecommunications networks from the Universitat Politècnica de València (UPV), Valencia, Spain. In 2016, he received his Ph.D. degree in telecommunications from UPV. Actually, he work at the Escuela Superior Politécnica de Chimborazo (ESPOCH), Riobamba, Ecuador. Also, he has worked in investigation and cooperation projects between ITEAM and the Colombian spectrum regulator (ANE). His research activities are focused on spectrum optimization, DTT network planning, compatibility studies between DTT and other mobile technologies as LTE, as well the implementation of $4 \mathrm{G}$ communications in the Digital Dividend. 